\title{
Stacking Multiple High Resolution Hard X-ray Zone Plates at 2-ID-E of the Advanced Photon Source
}

\author{
$\underline{\text { Michael Wojcik }}^{1, *}$, Olga Antipova ${ }^{1}$, Christian Roehrig $^{1}$, and Deming Shu ${ }^{1}$ \\ 1. X-ray Sciences Division, Argonne National Laboratory, Argonne, IL, USA. \\ * Corresponding author, mwojcik@anl.gov
}

Focused probe based beamlines at synchrotron sources perform a variety of microscopy techniques, recording different signals under a wide array of conditions on a wide array of samples. Generally, increasing flux in a smaller beam spot size will increase the output of the beamline. High quality focusing optics are key to and improvements to the optics have a direct benefit to the performance of the beamline. This need for high performance optics will become greater as new generation of synchrotron sources are turned on based on multi-bend achromat rings such as the Advanced Photon Source Upgrade (APS-U).

Hard X-ray zone plates are diffractive optics similar to circular diffraction gratings but with changing zone period as a function of radius. The diffraction limited resolution is determined largely by the outer zone width. Using state-of-the-art nanofabrication capabilities, zone width below $10 \mathrm{~nm}$ is possible [1]. The primary disadvantage of hard X-ray zone plates arises from the limited focusing efficiency of the diffractive optic. Effective efficiency is limited by the zone thickness, material, and X-ray energy focused; and is usually several microns thick for Hard X-ray energy. Achieving the high aspect ratio required for sub-100 nm outer zone width and zone height multiple microns thick is not currently possible without very specialized zone plate structures, such as $\mathrm{Si} /$ metal zone plate based on metal-assisted etching (MACE) [2] with their own accompanying disadvantages. Another strategy is to stack multiple zone plates in a specialized stacking device in the intermediate field [3]. In this paper, we will show results from stacking up to 4 zone plates with $40 \mathrm{~nm}$ outer zone width at the 2-ID-E microprobe of the APS.

Hard X-ray zone plates were fabricated at the Center for Nanoscale Materials (CNM), using a single layer electroplating mold fabrication process. Samples started as silicon nitride membranes with a Si frame and an electroplating layer on the working side. Poly (methyl methacrylate) (PMMA) was spun onto the working side to $400 \mathrm{~nm}$ thickness using MicroChem 950PMMA A5.5 resist Samples were exposed using a JEOL JBX-9300FS electron beam lithography system with up to $3000 \mu \mathrm{C} / \mathrm{cm}^{2}$ exposure dose at 100 $\mathrm{keV}$ beam energy in the zone plate pattern. After exposure, samples were developed in 4:1 ethanol:deionized water solution for 40 seconds, rinsed in pure ethanol, then dried with flowing $\mathrm{N}_{2}$ gas. Zones were formed by electroplating the resulting mold in Technic 25E Au-sulfite solution heated to 40 ${ }^{\circ} \mathrm{C}$ using a plating current density of $2 \mathrm{~mA} / \mathrm{cm}^{2}$ and time was estimated to fill the zones. Though in the case for three of the zone plates tested, the plating rate was greatly underestimated and the zones were partially filled to $150 \mathrm{~nm}$ and had a low individual efficiency. The PMMA mold was removed using Dow MICROPOSIT Remover 1165 and ready for use. The base zone plate had $240 \mu \mathrm{m}$ in diameter and 400 $\mathrm{nm}$ zone thickness, the other zone plates in the stack had diameters of $242.5,245$, and $247.5 \mu \mathrm{m}$ for a 0.8 mm separation between zone plates at $10 \mathrm{keV}$ X-ray energy.

Zone plates were taken to 2-ID-E at APS and mounted to the stacking device (figure 1) with 12 axes of freedom on 4 arms and one additional fixed arm with the beamstop mounted. Each zone plate was mounted on a $100 \mu \mathrm{m}$ thick, $5 \mathrm{~mm}$ wide, and $30 \mathrm{~mm}$ long diamond optics holders from Applied Diamond Inc. The beamline setup for 2-ID-E included an $80 \mu \mathrm{m}$ horizontal slit, a side bounce crystal to extract a portion of 
the insertion device beam, and mirror for harmonic rejection and alignment of the beam. In the station, an upstream $500 \mu \mathrm{m}$ pinhole apertures the beam, while downstream of the zone plates was a $30 \mu \mathrm{m}$ order sorting aperture, sample, and detectors. Both fly and step scans were available, due to speed fly scans were performed during focusing but a problem occurred causing the image scans to be done using step scanning. An auto tweak program was implemented to quickly align the zone plates in the transverse direction.

The total efficiency of all four zone plates stacked was $6.3 \%$ at $10 \mathrm{keV}$. The efficiency for the base zone plate was $2.7 \%$, while the other three zone plates each had $\sim 0.5 \%$ focusing efficiency with less than $0.1 \%$ variation between zone plates. The focus determined by knife edge scans in the horizontal plane was 170 $\mathrm{nm}$ for all four zone plates and approximately the same for the individual zone plates. A $100 \mathrm{~nm} \mathrm{Au}$ inner spoke Siemens star was imaged (figure 2) multiple times over a several hour period to test the stability of the setup and two images from the set of scans is shown.

We observed approximately a 10\% reduction in flux through the sample over a period of one hour, and around a $50 \%$ reduction over a 12 hour period. Simulations done using a wave propagation code [4] calculated a $10 \%$ reduction in flux with a single zone plate transverse misalignment of $15 \mathrm{~nm}$, close to $1 / 3^{\text {rd }}$ of the outer zone width. Correspondingly, we measured $\sim 10 \%$ reduction in flux with $10-20 \mathrm{~nm}$ misalignment found with the auto tweak program scans, but this is only an approximation. The stacking apparatus and zone plates were in air, cantilevered on an adapter L-bracket attached to large motion translation stages. Also, temperature in the hutch fluctuated by several degrees when the door to the hutch opened, though efforts was made to minimize temperature impact on stability. Further study must be done to improve the stability of the stacking apparatus, while the auto tweak program provides another possible path for improving the long term stability of stacked zone plates during image scans. [5]

\section{Reference:}

[1] Mohacsi, Istvan, et al., Sci. Reports 7 (2017), p. 43624.

[2] Li, Kenan, et al., J. of Vac. Sci. \& Technol. B, 35.6 (2017), p. 06G901.

[3] Gleber, Sophie-Charlotte, et al., Optics express 22.23 (2014), p. 28142-28153.

[4] Vila-Comamala, Joan, et al., J. of Synchrot. Rad. 20.3 (2013), p. 397-404.

[5] Use of the Center for Nanoscale Materials and the Advanced Photon Source, Office of Science user facilities, was supported by the U.S. Department of Energy, Office of Science, Office of Basic Energy Sciences, under Contract No. DE-AC02-06CH11357. We thank Ralu Divan, Leonidas Ocola, Liliana Stan, Bing Shi, and Stefan Vogt for their help during this research.

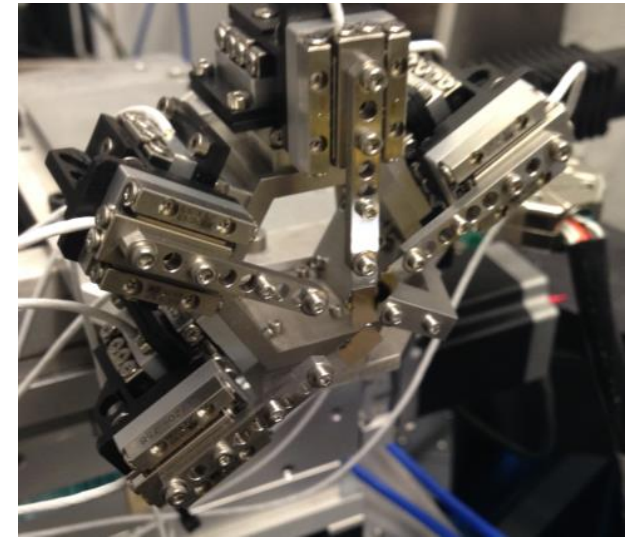

Figure. 1. Stacking apparatus with four arms with three axes and a fixed arm at 2-ID-E.
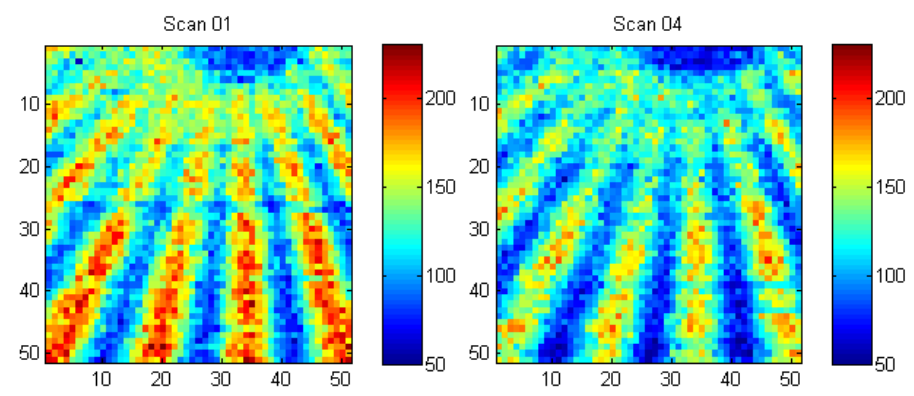

Figure. 2. Two fluorescence images of the Au $100 \mathrm{~nm}$ Siemens star taken 4 hours apart to show reduction of intensity. Scans were $51 \times 51$ points, $100 \mathrm{~nm}$ step size, and $1 \mathrm{sec}$ per point. 